\title{
Promoção de saúde bucal em um ambiente hospitalar infantil: um relato de experiência
}

\author{
Oral health promotion in a children's hospital environment: an experience report
}

Promoción de la salud bucal en un entorno hospitalario infantil: un informe de experiencia

Diego Costa Rodrigues ${ }^{1 *}$, Luiz Gabriel Pacífico Santos ${ }^{1}$, Waldo Silva Mariz ${ }^{1}$, José Lucas Medeiros Torres $^{1}$, Natercia Medeiros de Lima ${ }^{1}$, Igor Natanyo de Freitas Silva ${ }^{1}$, Rafaella Bandeira de Melo Souza Cavalcanti ${ }^{1}$, Rachel de Queiroz Ferreira Rodrigues ${ }^{1}$, Fátima Roneiva Alves Fonseca ${ }^{1}$, Maria Carolina Bandeira Macena ${ }^{1}$.

\section{RESUMO}

Objetivo: O objetivo deste trabalho é, por meio de um relato de experiência de alunos extensionistas do Programa "Heróis do Sorriso: humanizando olhares para um resgate de atitudes", ressaltar a importância da realização de promoção de saúde bucal em um ambiente hospitalar infantil, ressaltando a necessidade da prevenção da doença cárie, além de fomentar o cuidado e a autonomia com a saúde bucal. Ademais, objetivou-se esclarecer a importância da realização de visitas periódicas ao cirurgião-dentista. Relato de Experiência: Atividades recreativas e educativas foram elaboradas pelos universitários com crianças de idade entre 3 a 15 anos em um hospital infantil, do estado da Paraíba, durante o ano de 2019. O intuito do projeto foi tornar a condição de permanência da criança no hospital um pouco mais tolerável, distraindo o paciente em um momento tão difícil de enfermidade, além de fornecer conhecimento sobre saúde bucal. Considerações Finais: Ficou evidente que a vigência do projeto possibilitou aos graduandos de odontologia uma forma de trabalhar com práticas de saúde bucal fora do ambiente acadêmico, bem como atuar em atividades lúdicas que melhoram o processo educativo infantil acerca da saúde bucal, além de ajudar no condicionamento clínico e hospitalar das crianças, em práticas pontuais e lineares de prevenção e cuidado.

Palavras-Chave: Odontopediatria, Saúde pública, Humanização da assistência.

\section{ABSTRACT}

Objective: The objective of this work is, through an experience report of extension students from the "Heroes of the Smile: humanizing looks for a rescue of attitudes" program, to emphasize the importance of promoting oral health in a children's hospital environment, emphasizing the need to prevent caries disease, in addition to promoting care and autonomy with oral health. In addition, the objective was to clarify the importance of periodic visits to the dental surgeon. Experience Report: Recreational and educational activities were developed by university students with children aged 3 to 15 years in a children's hospital, of the state of Paraíba, during 2019. The purpose of the project was to make the condition of permanence of the child in the hospital a little more tolerable, distracting the patient in such a difficult moment of illness, besides providing knowledge about oral health. Final Considerations: It was evident that the duration of the project enabled dental students to work with oral health practices outside the academic environment, as well as to act in recreational activities that improve the children's educational process about oral health, in addition to helping in children's clinical and hospital conditioning, in specific and linear prevention and care practices.

Keywords: Pediatric dentistry, Public health, Humanization of assistance.

\section{RESUMEN}

Objetivo: El objetivo de este trabajo es, a través de un relato de experiencia de los estudiantes de extensión del programa "Héroes de la Sonrisa: humanizando busca un rescate de actitudes", enfatizar la importancia de promover la salud bucal en un ambiente hospitalario infantil, enfatizando la necesidad de prevenir la enfermedad de la caries, además de promover el cuidado y la autonomía con la salud bucal. Además, el

${ }^{1}$ Universidade Federal de Campina Grande (UFCG), Patos - PB. *E-mail: rafaella.bm@hotmail.com 
objetivo era aclarar la importancia de las visitas periódicas al cirujano dentista. Informe de Experiencia: Las actividades recreativas y educativas fueron desarrolladas por estudiantes universitarios con niños de 3 a 15 años en un hospital infantil, del estado de Paraíba, durante el 2019. El propósito del proyecto fue hacer la condición de permanencia del niño en el hospital un poco más tolerable, distrayendo al paciente en tan difícil momento de enfermedad, además de aportar conocimientos sobre salud bucal. Consideraciones Finales: Se evidenció que la duración del proyecto permitió a los estudiantes de odontología trabajar con prácticas de salud bucal fuera del ámbito académico, así como actuar en actividades recreativas que mejoren el proceso educativo de los niños sobre salud bucal, además de ayudar en la acondicionamiento clínico y hospitalario infantil, en prácticas específicas y lineales de prevención y atención.

Palabras Clave: Odontología pediátrica, Salud pública, Humanización de la atención.

\section{INTRODUÇÃO}

Sabe-se que a infância é um período de surgimento de muitas enfermidades, e na maioria das vezes a ida ao tratamento em ambiente hospitalar é inevitável, porém os procedimentos em hospitais apresentam para as crianças um caráter mais doloroso, invasivo e de medo, podendo acarretar prejuízo físico, psíquico e intelectual (PARCIANELLO AT e FELIN RB, 2008). Assim, as relações interpessoais e a comunicação efetiva com os pacientes são fatores determinantes da qualidade da atenção no atendimento e na melhoria da saúde (SHITSUKA C, et al., 2019).

O envolvimento do público infantil com os cirurgiões-dentistas é especialmente importante, devido ao fato de o paciente odontopediátrico apresentar necessidades muito distintas em comparação às dos adultos, devido a capacidade limitada dessas crianças quanto à adaptação ao ambiente clínico-odontológico (MCGILTON K, et al., 2006). Alguns estudos afirmam que os profissionais de odontologia têm desenvolvido, através da sua prática profissional, habilidades pessoais e estratégias de empatia para conseguir influenciar a experiência da criança e da sua família, tornando-a positiva para melhoria da saúde bucal do público infantil (LINDEKE L, et al., 2006).

No entanto, segundo a Pesquisa Nacional de Saúde Bucal, realizada no Brasil em 2010, apenas 46,6\% das crianças brasileiras com cinco anos e $43,5 \%$ das crianças com doze anos não apresentam a doença cárie, fato esse que na maioria das vezes é acarretado pela falta de informações sobre saúde bucal (BRASIL, 2012).

Paralelo a isso, foi observado que a falta de informações e atendimentos foram ainda mais intensas em locais de difícil acesso, tais quais hospitais, creches e as próprias escolas (ANTUNES C, 2006). Dessa forma, atividades realizadas pelos acadêmicos nesses ambientes, como no hospital infantil de um município no estado da Paraíba, pode favorecer o envolvimento da criança na construção de novos conhecimentos, facilitando a mudança de atitudes, hábitos e cuidados, além de ajudar a melhorar a harmonia local, visto que se está trabalhando com pacientes pediátricos que apresentam enfermidades (GHAFFAR M, et al., 2018; OLIVEIRA GG, 2011).

O processo de ambientação ao local hospitalar na infância é, sem dúvida, um momento marcante na vida de qualquer criança, uma vez que, neste período, ela passa por uma frágil adaptação durante a realização dos procedimentos, alterando a sua rotina diária, que antes envolvia brincar e ir à escola. A concepção da infância é intimamente ligada ao bem-estar, energia e alegria, o que torna mais difícil assimilar a doença e a hospitalização nesta fase do ciclo vital, tanto por parte da própria criança, como de toda sua rede de apoio (LINHARES MB, et al., 2015).

Todas essas mudanças podem causar um impacto na visão dos pequenos, podendo mudar seu comportamento durante e depois da internação. Assim, a hospitalização e o adoecimento podem ser fatores de risco para o desenvolvimento infantil (CECCIM RB e CARVALHO PRA, 2009). Nessa perspectiva, a imagem do profissional da área da saúde como auxiliador vem como ato ouro para garantir não apenas o bom atendimento, mas também para diminuição de traumas ao ambiente hospitalar e melhor adaptação das crianças. 
Sendo assim, o presente artigo possui como objetivo destacar a figura do cirurgião-dentista em ambientes característicos das referidas dificuldades, bem como reforçar a grande importância dos acadêmicos de odontologia como replicadores de informações que impulsionem a promoção de saúde e prevenção de doenças relacionadas à saúde bucal, principalmente em ambientes com pouco acesso, auxiliando, dessa forma, na melhoria do condicionamento hospitalar e clínico das crianças e no fortalecimento da autonomia e cuidados com o público infantil.

\section{RELATO DE EXPERIÊNCIA}

O hospital Infantil trabalhado corresponde a um centro hospitalar único do sertão paraibano, dedicado exclusivamente ao atendimento público infantil. Além disso, o hospital é referência para mais de 40 cidades polarizadas ao redor do município no qual está localizado, tendo contato com as fronteiras de outros estados. O centro hospitalar conta com mais de 300 atendimentos diários, sendo composto por 60 leitos pediátricos, distribuídos em 5 enfermarias clínicas, 1 isolamento clínico, 1 enfermaria cirúrgica, 6 leitos de UTI pediátrica, sendo um com isolamento e um bloco cirúrgico com uma sala operatória.

Ademais, o hospital conta com a presença de uma Sala de Recreação, tendo como tema "O Pequeno Príncipe", sendo esta sala completamente equipada de brinquedos, materiais didáticos e áudios-visuais, o que proporciona maior entretenimento das crianças no período em que estiverem internadas ou aguardando 0 atendimento médico.

A experiência aqui relatada foi realizada com crianças que possuíam idade entre 3 e 15 anos e que estavam internadas no referido centro médico, no ano de 2019. O eixo do hospital do programa de extensão "Heróis do Sorriso" contava com a participação de 8 integrantes, sendo: 1 professora coordenadora, 2 alunos bolsistas e 5 alunos voluntários.

O seu principal objetivo foi tornar a condição de permanência da criança no hospital um pouco menos sofrida e mais tolerável, distraindo o paciente em um momento tão difícil de enfermidade, além de fornecer conhecimento sobre saúde bucal. Devido às circunstâncias, a maior dificuldade apresentada era a condição em que a criança se encontrava, portanto, procurou-se estabelecer um contato direto com os responsáveis do hospital e equipe médica a fim de respeitar as particularidades e necessidades de cada enfermo, garantindo a segurança de todos.

As ações foram realizadas semanalmente, especificamente às sextas-feiras, e eram previamente organizadas e planejadas pelo grupo, com o intuito de sempre diversificar os temas abordados. Dentre eles se destacavam doença cárie, traumatismo dentário, bons e maus hábitos, técnicas de escovação e dieta, pois são problemas de maior incidência em atendimentos odontopediátricos. Através de dinâmicas, linguagem e materiais lúdicos e educativos se conseguia abordar tais assuntos com as crianças e simultaneamente divertilas nesse momento estressante.

Com o propósito de ganhar a atenção das crianças os integrantes do projeto se fantasiavam de superheróis durante as ações, com pinturas, máscaras e utilizando jalecos coloridos, sempre com um bom-humor para distribuir alegria a todos, diminuindo a ansiedade dos pacientes e obtendo a confiança destes através de conversas, oferecendo uma bexiga ou chamando para brincar, fazendo com que a criança se sentisse mais à vontade e tranquila.

De acordo com o tema escolhido para se trabalhar no dia, o grupo planejava e confeccionava materiais anteriormente à ação. Estes tinham caráter lúdico, eram chamativos e despertavam curiosidade nos pacientes. Bons exemplos destes aparatos utilizados eram: o macro modelo de uma boca para auxiliar durante a instrução de higiene oral, um dente exibindo o processo de evolução da cárie para facilitar o entendimento da criança, além de uma cesta de alimentos confeccionados com feltro para contribuir nas explicações sobre dieta cariogênica, entre outros.

Durante os encontros um grupo de extensionistas se responsabilizava em atender pais e responsáveis, instruindo-os sobre a importância da manutenção da saúde bucal infantil e da influência da mesma sobre a recuperação da saúde sistêmica, também esclarecendo dúvidas sobre o atendimento odontológico pediátrico e encaminhando-os, se necessário, ao atendimento da clínica de atendimento odontológico. 
Por se tratar de um ambiente hospitalar havia uma preocupação com a situação em que as crianças se encontravam, tendo em vista suas limitações físicas. Dessa forma, era preferível a realização de atividades que não necessitassem de movimentação e que se adequassem às condições psicológicas e às necessidades do público-alvo. Bons exemplos são o jogo do "dente feliz e dente triste" e o jogo da memória, que eram bastante utilizados nas atividades. O primeiro consistia em mostrar figuras de hábitos bons e ruins para saúde bucal e questionar a opinião das crianças, encaixando a figura na boca saudável ou doente. No jogo da memória, feito com cartas que representavam alimentos e hábitos de higiene oral, a criança, ao encontrar o par, era instigada a dialogar sobre o conteúdo do mesmo, aprendendo, dessa maneira, condutas benéficas.

Eram ensinadas técnicas de escovação dentária no manequim odontológico, tanto para as crianças, quanto para os respectivos pais, responsáveis e funcionários, explicando a importância da utilização do kit de higiene oral, o qual consiste em uma escova de dente, um dentifrício fluoretado e um fio dental. Ao final de todas as brincadeiras e tarefas as crianças recebiam pequenas premiações, como: desenhos educativos para colorir, balões, pinturas com tinta guache no corpo, modelo de "certificado de bom paciente" e outros, para que isso ajudasse no reforço positivo, no incentivo da disseminação de informações e na prática dos bons hábitos aprendidos.

Em certas datas comemorativas foram elaboradas ações que pudessem levar alegria ao ambiente hospitalar e que permitissem que as crianças pudessem comemorá-las, como é o caso do dia das crianças, no qual os extensionistas se fantasiaram e distribuíram brinquedos para os pacientes, desenvolvendo também dinâmicas adaptadas a cada limitação, prezando sempre pela participação de todos no momento.

Outra ocasião especial foi o "São João do Hospital Infantil" onde todos foram vestidos com trajes juninos, além de confeccionarem acessórios a caráter para os pacientes e brincadeiras temáticas de festas juninas, como "Pescaria" e "Jogo das argolas", todos adequados para que as crianças pudessem participar sem necessitar de muito esforço. Promover saúde bucal em um ambiente tão complexo como um Hospital Infantil não é uma tarefa simples, mas conseguir melhorar o dia de uma criança enferma, receber um sorriso sincero e auxiliá-la em um momento tão delicado é certamente gratificante.

Para os graduandos extensionistas, essa experiência proporcionou aprendizados em diversos aspectos, onde foi possível aplicar os conhecimentos odontológicos adquiridos em sala de aula e na clínica escola, levando-os para comunidade e promovendo saúde bucal, além de poder melhorar a estadia das crianças assistidas, podendo Ihes proporcionar alegria, além de gratidão nos responsáveis que as acompanhavam. Todo esse processo contribui para a formação de profissionais mais sensíveis e empáticos, colaborando para construção de uma Odontologia humanizada, mais abrangente e menos limitada a procedimentos clínicos.

\section{DISCUSSÃO}

Os programas de promoção de saúde bucal objetivam sempre a maior qualidade de vida dos indivíduos, para isso são realizadas ações de prevenção sendo estas essenciais para um futuro controle adequado (MARTINS CR, et al., 2011). Seguindo essa premissa, o eixo do Hospital do programa de extensão universitária "Heróis do Sorriso" procurou inserir este objetivo em suas atividades, atuando com sensibilidade, respeitando as limitações e necessidade das crianças atendidas durantes as ações desenvolvidas no hospital infantil de um município do estado da Paraíba.

O ambiente hospitalar não deve ser encarado apenas como um ambiente de práticas de cura e reabilitação, deve ser um espaço de promoção da saúde, de defesa da vida e da cidadania, com atuação de todos envolvidos e a comunidade na formação de novas relações dentro do sistema de saúde (SILVA MM, et al., 2010).

Ao pesquisar e criar sempre atividades lúdicas e recreativas que se adequem àquela realidade encontrada, transformando as crianças hospitalizadas em verdadeiros protagonistas de histórias com finais felizes, a maior meta do programa de extensão Heróis do Sorriso é atingida e evidenciada pelos próprios profissionais e pais presentes, que relatam uma maior adesão aos tratamentos, bem como a melhora significativa do humor da criança, muitas vezes abalado pelas rotinas de exames, dor e pela experiência de ter que ficar longe dos familiares. 
A maior dificuldade encontrada pelo programa são as particularidades e limitações de cada criança hospitalizada. Para que estas dificuldades fossem superadas, em cada visita ao hospital eram levadas cerca de três a quatro atividades diferentes e a maioria delas eram desenvolvidas com as crianças acamadas com impossibilidade de mover uma das mãos em decorrência das medicações intravenosas, visando sempre a necessidade de cada enfermo e a segurança de todos.

Sendo a saúde fortemente influenciada por diversos fatores socioeconômicos, culturais, físicos e ambientais, as ações em prol da promoção de saúde bucal visam aumentar as oportunidades de prevenção dos agravos que estas doenças trazem e que impactam diretamente na qualidade de vida dos indivíduos (ROSA J, et al., 2010).

O programa de extensão Heróis do Sorriso busca diante desse cenário internalizar através de suas ações educativas uma maior consciência da responsabilidade individual no processo de obtenção de uma melhor qualidade de vida daqueles que não possuem assistência adequada dos programas de saúde (SILVA GG, et al., 2017). O constante interesse dos alunos do curso de odontologia da Universidade Federal de Campina Grande e o elevado número de inscrições durante as seleções para programa denota que este objetivo vem sendo atingido.

Entende-se nesse sentido que as atividades de extensão desenvolvidas pelo programa Heróis do Sorriso acrescentam tanto como campo da extensão, quanto para atividades de ensino. A interligação universidade e comunidade faz parte do processo cultural e científico, unindo o ensino e extensão de forma inseparável e contribuindo então para formação de um profissional da saúde mais comprometido com as causas sociais e promoção de saúde (OLIVEIRA JS, et al., 2015).

Em suma, é notável o impacto positivo que o programa de extensão Heróis do Sorriso vem causando na vida dos alunos de graduação em diversos quesitos e também dos membros da comunidade assistida, sendo imprescindível a sua progressão para que o vínculo estabelecido entre a comunidade e a universidade seja cada vez mais fortalecido. Entretanto, para que as ações de promoção de saúde desenvolvidas pelo programa tenham êxito é necessário o comprometimento e dedicação de todos envolvidos, desde as etapas de planejamento, confecção de materiais e presença no dia das ações.

\section{REFERÊNCIAS}

1. ANTUNES C. A linguagem do afeto: como ensinar virtudes e valores. Campinas: Papirus, 2006.

2. BRASIL. Ministério da Saúde. Secretaria de Atenção à Saúde. Secretaria de Vigilância em Saúde. SB Brasil 2010 : Pesquisa Nacional de Saúde Bucal: resultados principais. Brasília, 2012.

3. CECCIM RB, CARVALHO PRA. Criança hospitalizada: A atenção integral como uma escuta à vida. Porto Alegre: Ed. UFRGS; 2009.

4. GHAFFAR M, et al. Oral Health Education and Promotion Programmes: Meta-Analysis of 17-Year Intervention. International Journal of Dental Hygiene, 2018, 16(4): 59-67.

5. LINDEKE L, et al. Capturing chilndren's voices for quality improvement. Am J Matern Child Nurs, 2006, 31(5): 290295.

6. LINHARES MB. Psicologia Pediátrica: aspectos históricos e conceituais. In: Anais do VII Ciclo em Saúde Mental. Ribeirão Preto, 2015.

7. MARTINS CR, et al. Projeto "Sorrindo na Escola": relato de experiência do programa na motivação e promoção da saúde bucal em escolares. Rev Odontol UNESP, 2011, 40: 18.

8. MCGILTON K, et al. Communication enhancement: nurse and patient satisfaction outcomes in a complex continuing care facility . J Adv Nurs, 2006, 54(1): 35-44.

9. OLIVEIRA GG. Neurociências e os processos edicativos:um saber necessário na formação de professores. Dissertação de Mestrado. Universidade de Uberaba, Minas Gerais, 2011; $147 \mathrm{p}$.

10. OLIVEIRA JS, et al. Promoção de saúde bucal e extensão universitária: novas perspectivas para pacientes com necessidades especiais. Revista Abeno, 2015, 15(1), 63-69.

11. PARCIANELLO AT, FELIN RB. E agora doutor, a onde vou brincar? considerações sobre a hospitalização infantil. Barbaroi, 2012, 28, 147-166.

12. ROSA J, et al. A Sala de Espera no Agir em Saúde: Espaço de Educação e Promoção á Saúde. Perspectiva Erechim, 2010, 35(129), 121-130.

13. SANTOS LGP, et al. Manejo do comportamento infantil e saúde bucal na sala de espera: um relato de experiência. Research, Society and Development, 2020, 9(7): e888975069.

14. SHITSUKA C, et al. Influência dos pais sobre o comportamento infantil no atendimento odontológico. Res., Soc. Dev. 2019, 8(7): e43871154.

15. SILVA GG, et al. Estudo qualitativo sobre um programa de educação em saúde bucal. Cadernos Saúde Coletiva, 2017, 25(1), 7-13.

16. SILVA MM, et al. Promoção da Saúde em ambientes hospitalares. Rev Bras Enferm, 2010, 64(3): 596-599. 